\title{
Effect of the distribution of the arrivals and of the intermodal unit sizes on the transit time through freight terminals
}

\author{
G. Malavasi, A. Quattrini \& S. Ricci \\ D.I.T.S. Transport Area, University of Rome "La Sapienza", Italy
}

\begin{abstract}
The railway freight terminals play a key role within the multimodal logistic chain. Therefore, the transit time through these terminals represents one of the most relevant terminal performances and at the same time a key component of the freight transport generalized cost, so that its quantitative analysis is a strategic activity, both in the terminal planning (for the existing and for the future terminal) and in the logistic chain organization. The transit time is composed of deterministic and stochastic components, which increases significantly the problem complexity. The authors developed an original model based on queuing theory allowing the calculation of the transit time for a large variety of terminals. Some pilot applications of the model showed that the arrival distributions of both classes of vehicles are critical inputs for the effectiveness of the results. In this framework the present paper focuses on the theoretical study of these distributions including an extended validation campaign based on data available for an Italian case study (intermodal terminal of Pomezia near Rome). Another relevant aspect, affecting the quality of the model results, is the presence in the terminal of different sizes of intermodal units. In the paper a methodology to take into account these size differences effectively is also explained.
\end{abstract}

Keywords: intermodal, freight, terminal, transport unit, arrival distribution.

\section{Introduction}

The authors developed an original model [2-4] based on the queuing theory allowing the calculation of the transit time (TTR) of the freight transport units through the intermodal terminals. 
In this framework a refining of some key parameters of such model has been performed. After a synthetic description of the model structure, a methodological approach based on real collected data taking into account the influence of the intermodal unit size is exposed.

\section{Methodological approach}

The first step is to adopt a common transit time definition. Here is adopted the following one: "time period from the arrival of the single freight unit to the terminal gate from an external transport infrastructure to its exit from the terminal towards a different transport infrastructure".

The second step is the formalization of the model finalised to the determination of the transit time. With this purpose the following general and, nevertheless, simple formalization has been selected:

$$
T T R=T E(C, O)+T I(T, D, R)
$$

where:

- $\quad T E$ depends upon external infrastructures and transport services, to be formalized in two sets of parameters and constraints:

1. carrying capacity $C$ (e.g. railway line bottlenecks, etc.)

2. operation planning $O$ (e.g. timetable structures of the transport services arriving to the terminal and departing from it, etc.);

- $\quad T I$ depends upon technologies, dimensions and operational rules in the terminal, to be formalized in three sets of parameters and constraints:

1. terminal planning parameters $T$ (e.g. check-in and transfer technology);

2. terminal dimensions $D$ (e.g. distances between gates and transfer area, number of tracks, etc.);

3. operational constraints, rules and regulations $R$ (e.g. speed limits, maximum loading weights, etc.).

In order to define a common sequence of operations to be performed within the terminals, the single activities have been analyzed into details.

For each activity have been identified:

- $\quad$ an Operational Phase $(O P)$ and a previous Waiting Phase $(W P)$;

- $\quad$ the corresponding durations, Operation Time (TO) and Waiting Time $(T W)$.

The following list shows the single phases which have been identified for the most general cases:

1. Waiting before entering the terminal + Entering movement;

2. Waiting before check-in + Check-in operations;

3. Waiting before the first units transfer + First units transfer;

4. Waiting before the second unit transfer + Second units transfer;

5. Waiting before check-out + Check-out;

6. Waiting before exiting the terminal + Exiting movement.

In each generic terminal two different classes of entering and exiting vehicles ( $V^{\prime}$ and $V^{\prime \prime}$ ) may be identified. 
In the most general case $V^{\prime}$ and $V^{\prime \prime}$ may allow the transport of a very different amount $\left(N U^{\prime}\right.$ and $\left.N U^{\prime \prime}\right)$ of freight units. For instance in the land freight interchange is $N U^{\prime}$ (truck) < $<U^{\prime \prime}$ (train). Accordingly, in each terminal opposite flows of freight units entering on $V^{\prime}$ or $V^{\prime \prime}$ and exiting correspondingly on $V^{\prime \prime}$ or $V^{\prime}$ may be identified.

\section{Transit time calculation by model}

In figure 1 the duration of the single phases and the mean total transit times for the freight units running in both the directions (TTR' and $\left.T T R^{\prime \prime}\right)$ are represented. In the scheme of this figure a generic freight unit entering on a vehicle $V^{\prime}$ runs on it towards the transfer area and, after the stocking phase, proceeds on the vehicle $V^{\prime \prime}$. Therefore, in the most general case, the unit is transferred twice: from $V^{\prime}$ to the stocking area and from there to $V^{\prime \prime}$. Whenever a direct transfer from $V^{\prime}$ to $V^{\prime \prime}$ is possible, the second transfer phase $(O P 4)$ is not performed and correspondingly times (TO4 and TW4) are equal to 0 .

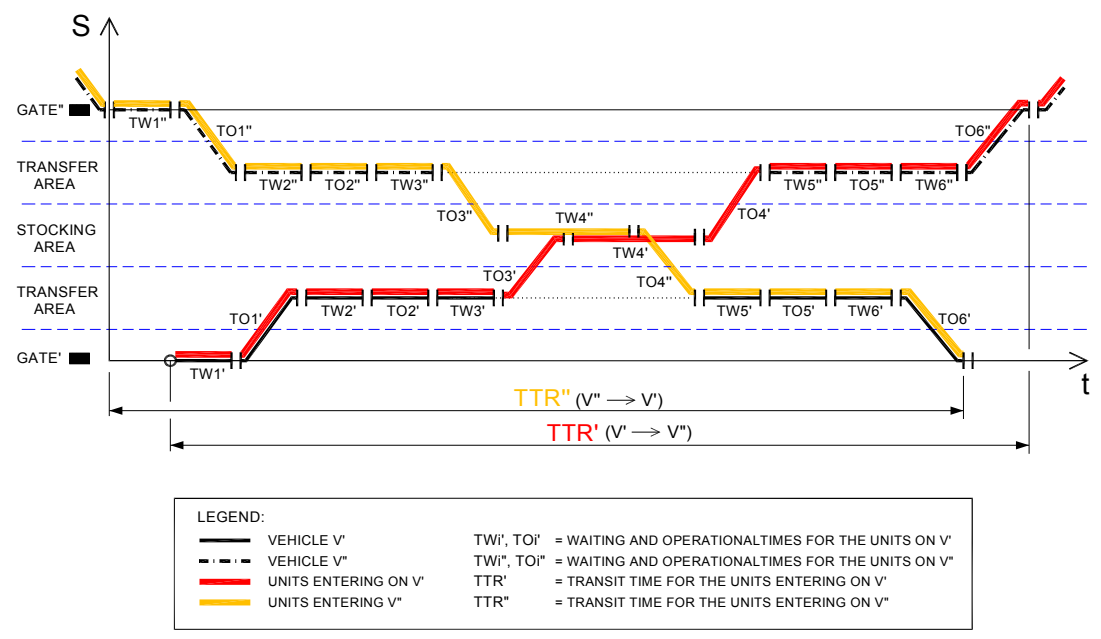

Figure 1: Single phases duration and total transit time in a generic terminal.

According to the unit flows within the plant represented in figure 1, the transit time may be formalized as follows:

$$
\begin{aligned}
& T T R^{\prime}=\sum_{i=1}^{4} T W_{i}^{\prime}+\sum_{i=1}^{4} T O_{i}^{\prime}+\sum_{i=5}^{6} T W_{i}^{\prime}+\sum_{i=5}^{6} T O_{i}^{\prime \prime} \\
& T T R^{\prime \prime}=\sum_{i=1}^{4} T W_{i}^{\prime}+\sum_{i=1}^{4} T O_{i}^{\prime \prime}+\sum_{i=5}^{6} T W_{i}^{\prime}+\sum_{i=5}^{6} T O_{i}^{\prime}
\end{aligned}
$$


Eqn. (2) represents the TTR for the freight units entering on $V^{\prime}$ and exiting on $V^{\prime \prime}$; eqn. (3) represents the TTR for the opposite flow (units entering on $V^{\prime \prime}$ and exiting on $\left.V^{\prime}\right)$.

\section{Validation campaign}

\subsection{Data collection}

The above-described generalized model has been applied to an Italian case study: the Pomezia freight intermodal terminal, located about $30 \mathrm{~km}$ south of Rome.

The authors collected a large quantity of data related to the transport units entering/exiting in/from the terminal on the two transport modalities. These data allowed the calibration of some key parameters to optimize the model formulation; moreover the study of the unit arrival/departure distribution is necessary to determine important input of the model (e.g. the mean time between 2 arriving trucks/trains).

In this context the paper focuses on the transfer of the units entering from the road side (by trucks $-V^{\prime}$ ) and exiting on the railway side (by trains $-V^{\prime \prime}$ ) of the terminal for the $T T R^{\prime}$ value calculation.

The following sections show the analysis performed on the collected data and its results.

\subsection{Arrival/departure distributions}

Figure 2 shows a graphic view of the collected data: the $x$-axis represents the terminal functioning period, the $y$-axis represents the number of units arriving to the terminal by truck (various colours correspond to different investigated days). Similar investigations have been carried out also for unit departing by truck.

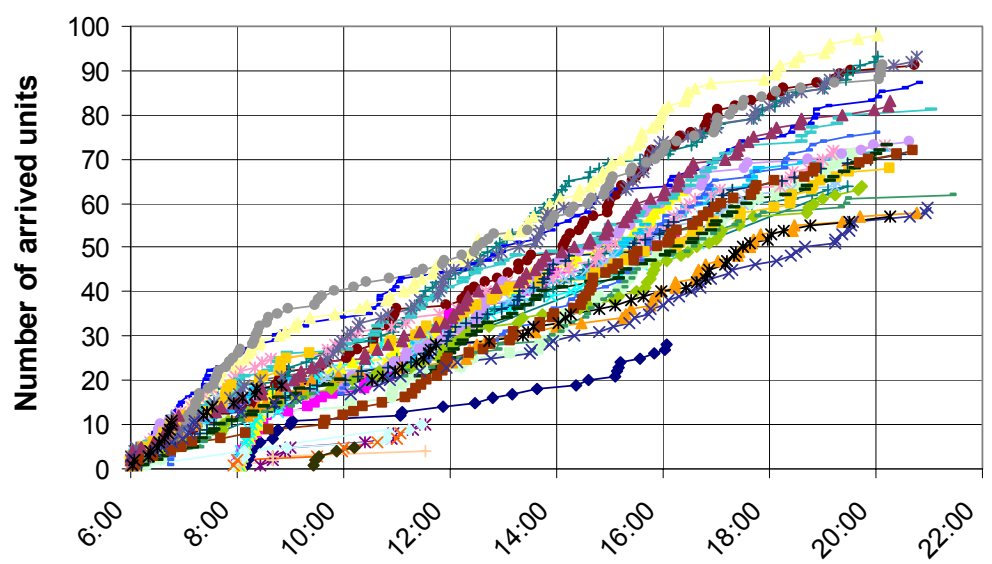

Unit arrival time

Figure 2: Units arriving by truck to the Pomezia terminal - collected data. 
The collected data have been elaborated to calculate the typical operational range, in which the most part of the real cases are included. The results of such elaboration are shown in figure 3 .

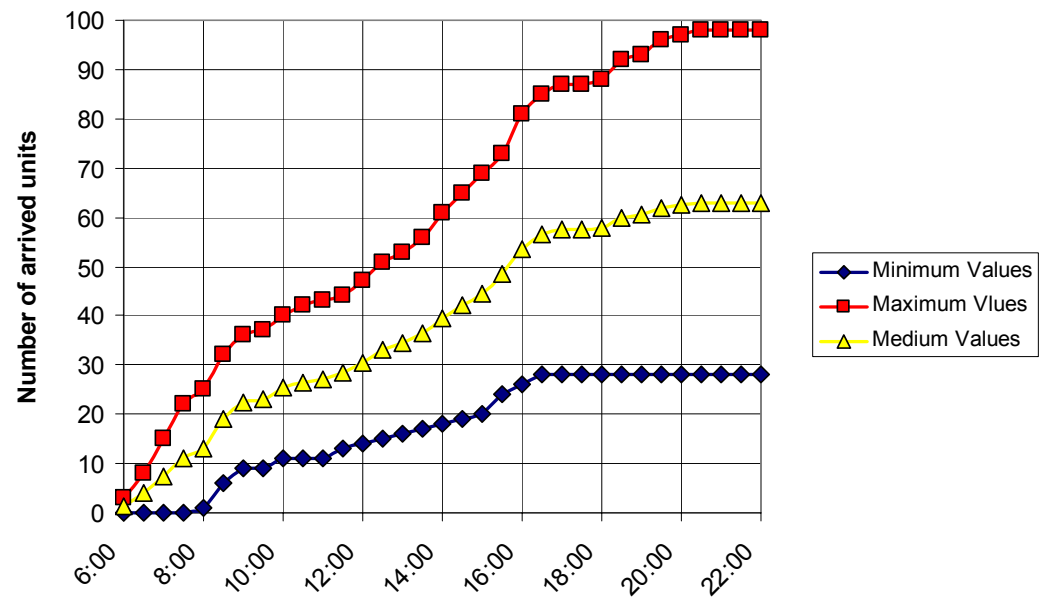

Unit arrival time

Figure 3: Distribution of units arriving on trucks - Pomezia Terminal.

The two curves of the figure have been obtained considering extreme and medium values occurring during the reference period.

- Similar analyses have been performed for the data collected on the train side. The results of such operations are shown in figure 4 .

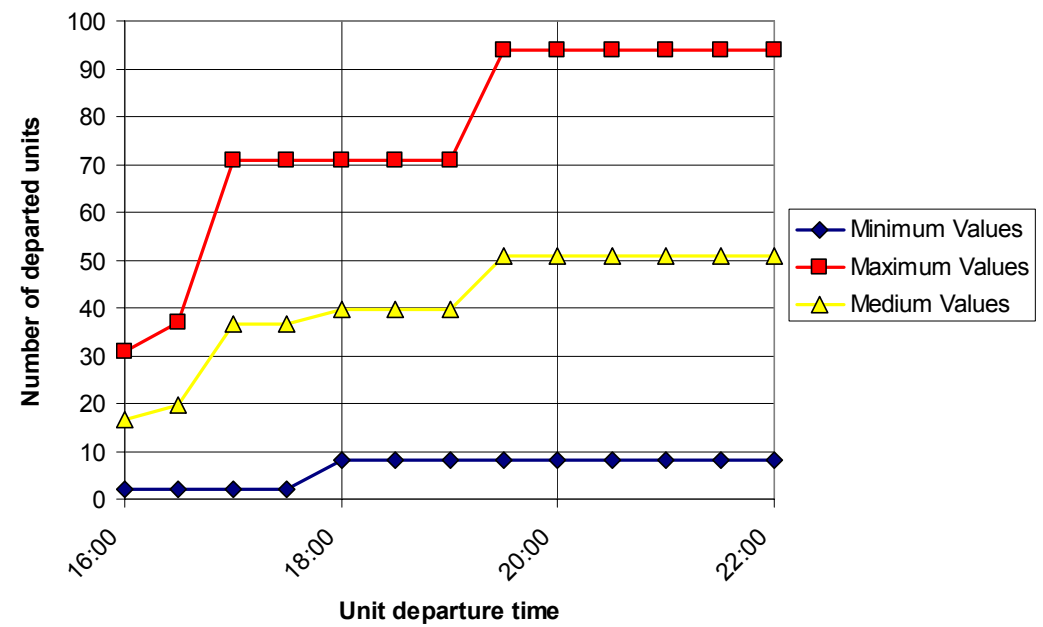

Figure 4: Distribution of units departing by train - Pomezia terminal. 
In the figure 4 case, the shape of the curves is quite different from the previous figure 3 . This is due to the fact that the number of trains departing from the terminal is largely lower than the number of arriving trucks.

Finally, the procedure shown in figure 5 has allowed the $T T R^{\prime}$ evaluation on the basis of the above-reported results (medium values graphically displayed by the yellow curves in figures 3 and 4 ).

The results obtained for the Pomezia terminal are the following:

$$
\begin{array}{ll}
- & T T R^{\prime}(\min )=3 h 30^{\prime} ; \\
- & T T R^{\prime}(\max )=8 h 00^{\prime} ; \\
\text { - } & T T R^{\prime}(\text { med })=5 h 45^{\prime} .
\end{array}
$$

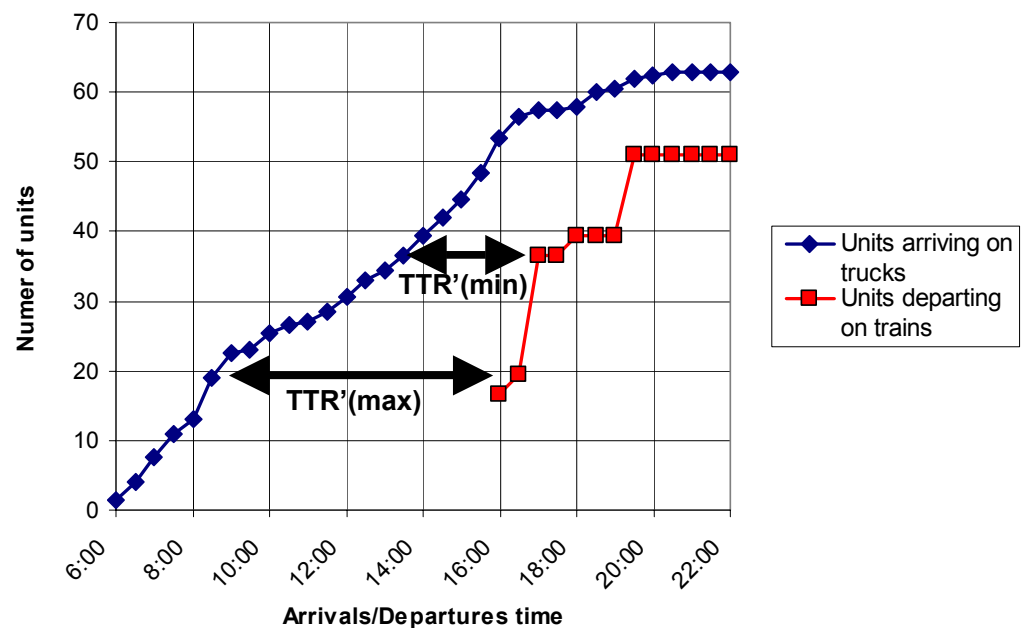

Figure 5: Graphic representation of $T T R^{\prime}(\max )$ and $T T R^{\prime}(\min )$ - Pomezia Terminal.

\subsection{Unit size variability}

Another aspect affecting the sensitivity of the model results is the presence in the terminal of different sized transport units; in fact with the same quantity of Twenty feet Equivalent Unit (TEU) is possible to handle a different number of intermodal transport units.

The EIA (European Intermodal Association), on the basis of extended investigations, suggests the following conventional equivalence between $T E U$ and ITU:

$$
1,4 \cdot I T U=2,3 \cdot T E U \Rightarrow 1 \cdot I T U=1,6 \cdot T E U
$$

On this basis, figure 6 shows two typical situations that can occur in the terminal: case $b$ (corresponding to the EIA conventional value) is easier to work because the transhipping device can transfer the same freight quantity with less handled units than in case $a(100 \% \mathrm{TEU})$. 
Accordingly to these observations, the TTR is expected to decrease when the $T E U / I T U$ ratio increases.

Case a: 5 Units $=5$ T.E.U.

\begin{tabular}{|l|l|l|l|l|}
\hline Unit 1 & Unit 2 & Unit 3 & Unit 4 & Unit 5 \\
\hline $20 \mathrm{ft}, 20 \mathrm{ft}, 20 \mathrm{ft}, 20 \mathrm{ft}, 2 \mathrm{ft}$ \\
\hline
\end{tabular}

Case b: 3 Units $=5$ T.E.U.

\begin{tabular}{|c|c|c|}
\hline Unit 1 & Unit 2 & Unit 3 \\
\hline $40 \mathrm{ft}, 20 \mathrm{ft}$ \\
\hline
\end{tabular}

Figure 6: $T E U-I T U$ correspondence.

\section{Case study application}

Figure 7 is a graphic view of the model application results to Pomezia terminal on the basis of the collected data.

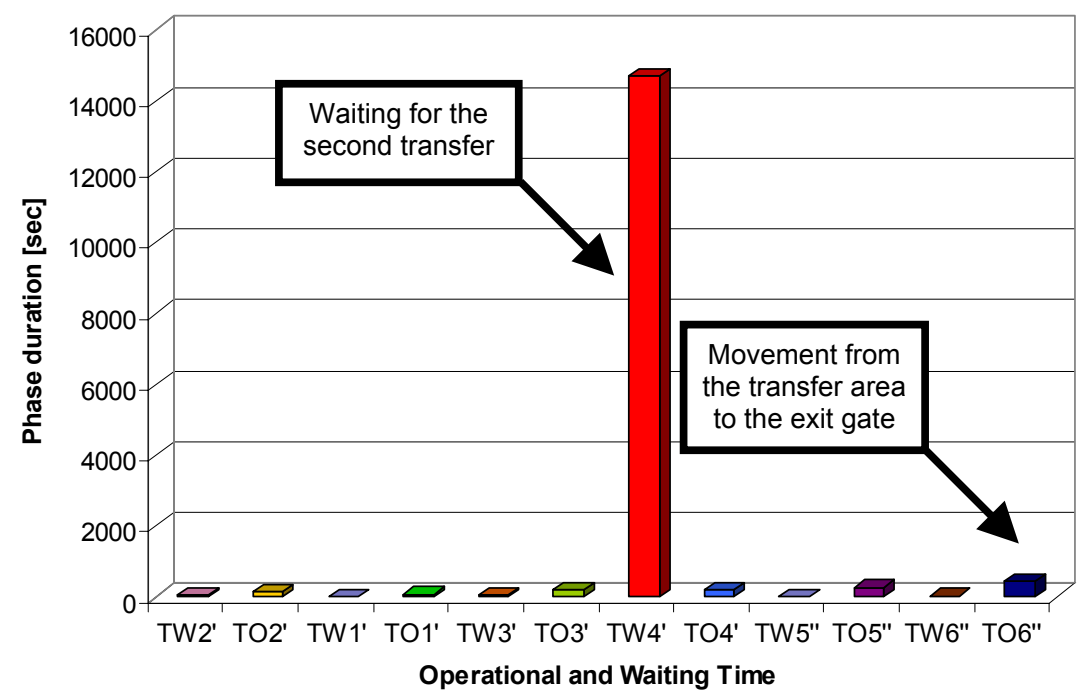

Figure 7: Model application results - Pomezia Terminal. 
The analysis of the numerical values of the single time components calculated by the model leads to the following considerations:

- $\quad$ calculated $T T R^{\prime}$ value is about $4 h 30^{\prime}$, so it provides a good representation of the real Pomezia situation shown in figure 5;

- for the units entering by trucks, the waiting for the second transfer in the stocking area $\left(T W 4^{\prime}\right)$ is largely the most important period within the terminal (about $91,6 \%$ of the global transit time);

- other important time period is due to the transport unit movement from the transfer area to the exit gate (it depends on the long distance that the unit must go through);

- in case of direct transfer from the first to the second vehicle (without stocking phase: $T W 4^{\prime}=T O 4^{\prime}=0$ ) the global transit time is strongly reduced;

- the internal parameters have a very limited influence on the global transit time;

- $\quad$ the waiting times are largely higher $\left(92 \%\right.$ of $\left.T T R^{\prime}\right)$ than the operational ones.

Figure 8 shows the $T T R^{\prime}$ sensitivity to the TEU/ITU ratio variation. The figure indicates an appropriate reduction of the $T T R^{\prime}$ values when the ratio increases (less handled transport units with the same freight quantity).

Anyhow, on the basis of the model results, the $T T R^{\prime}$ decreases extremely slowly; this is due to the external parameters that have a stronger effect than the internal ones on the global transit time value. Therefore, the operational activities of the terminal weakly affects the $T T R^{\prime}$ value.

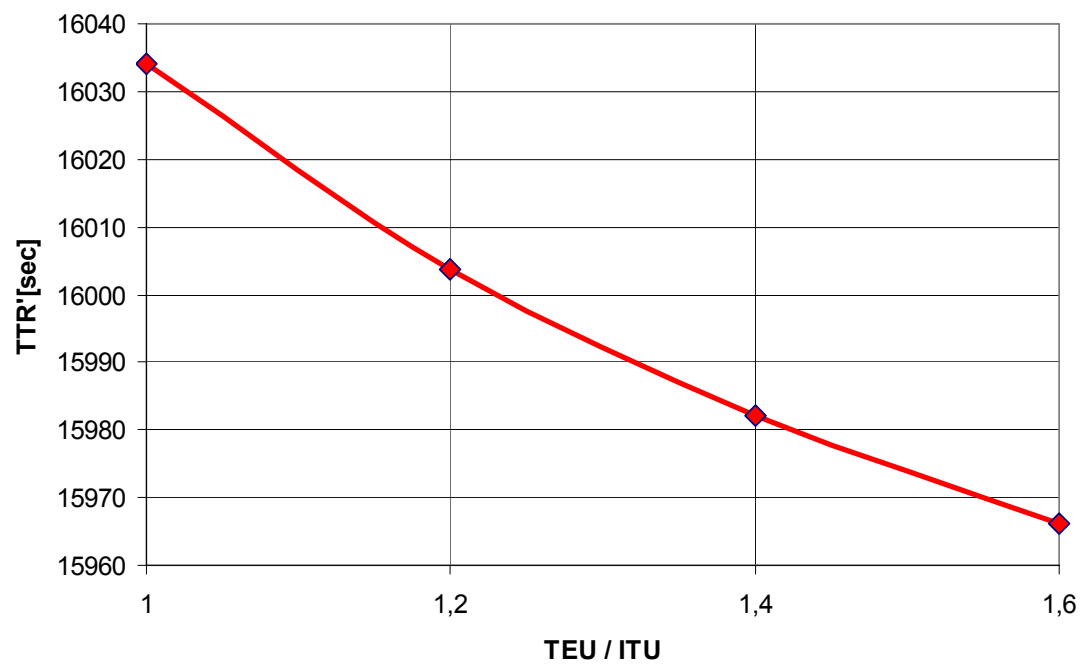

Figure 8: $\quad T T R^{\prime}$ sensitivity analysis to the $T E U / I T U$ ratio variation. 


\section{Conclusions and future research developments}

The developed model is characterized by wide generality and applicability to different terminal typologies, lay-outs, dimensions and transfer technologies.

The approach allows to highlight contributions and weights of the various activities and phases of the freight unit transit through the terminal by distinguishing operational and waiting periods, whose duration depends not only upon internal performances (technologies, dimensions and operational rules) but also upon external parameters and constraints (time distribution of vehicles arrivals and departures).

Therefore, this model can be widely helpful in the following application fields:

- Design of new terminals in the following design phases:

1. New terminal dislocations

2. Pre-dimensional study

3. Functions dislocations

4. Transhipping device choice

- Optimization of existing terminals by identifying the existing bottlenecks.

At present, the results of the application to some case studies show that the amount of time dedicated to the activities only depending upon internal parameters is generally very limited (about $5 \%$ in the land freight interchange case studies).

According to these results and to the present state of development, the research is going to be further developed in the following directions:

- consolidation and tuning of the methodological approach by means of a wider set of case studies applications based on the comparison with experimental data;

- model explication finalised to the systematic identification of internal and external carrying capacity bottlenecks;

- integration of the proposed model within a wider procedure which leads to the calculation of the total transport time from the origin to the final destination of the goods.

The performed analyses highlight that the application of the model to terminals which handle larger quantities of transport units is extremely important for a further model validation. These additional applications are a basic element to better evaluate the model results particularly in case of units entering by train (smaller quantity of events). Consequently, the authors are planning to extend the model application to a greater number of large and much exploited terminals.

\section{References}

[1] Arnold, D., Rall, B., Analyse des Lkw-Ankunftsverhaltens in Terminals des Kombinierten Verkehrs, Internationales Verkehrswesen 6, 1998.

[2] Malavasi, G., Ricci, S., A synthetic model for the evaluation of sea-side port terminal capacity. Proc. of the International Workshop of the Special 
Interest Group on Maritime Transport and Ports (SIG-2), World Conference on Transport Research Society, Genoa 2000.

[3] Malavasi, G., Ricci, S., Generalized model for the performance evaluation of different railway freight terminals. Proc. of the 9th International Symposium Zel 2002 - Railways on the Edge of the 3rd Millennium, University of Zilina - CETRA, Zilina 2002.

[4] Malavasi, G., Quattrini, A., Ricci, S. Personal communication, 18 november 2005, Urbanpromo 2005 Scientific Seminar - "Interporti, scali merci, centri logistici e sviluppo competitivo", Venice, IT.

[5] Malavasi, G., Zanolin, S., Formazione di treni merci e transito negli impianti di smistamento, Ingegneria Ferroviaria 8, 1997.

[6] RAIL Cargo Austria (Company). Cargo terminal saves space. Railway Gazette International Vol. 159 Issue 3, March 2003.

[7] Vandeveer, D., Intermodal rail facility design for the next century. Transportation Research Circular 459, June 1996.

[8] Verdon, L., Rousse, R., A menagement des gares terminales pour containers et equipements de manutention, Rail International 3, 1971.

[9] Zimmer, R.N., Designing intermodal terminals for efficiency. Transportation Research Circular 459, June 1996. 